\title{
Variabilité interraciale de la sensibilité à la rouille courbeuse chez le pin maritime
}

\author{
ML Desprez-Loustau 1, P Baradat 2 \\ 1 INRA, centre de Bordeaux, station de pathologie végétale, \\ BP 81, 33883 Villenave d'Ornon Cedex; \\ 2 INRA, laboratoire de génétique et amélioration des arbres forestiers de Bordeaux-Cestas, \\ 33610 Cestas, France
}

(Reçu le 3 décembre 1990; accepté le 28 juin 1991)

\begin{abstract}
Résumé - La sensibilité à la rouille courbeuse (Melampsora pinitorqua) de 16 provenances (appartenant à des races géographiques différentes) et hybrides interprovenances (entre la provenance landaise et une autre race géographique) de pin maritime a été étudiée en conditions d'infection naturelle dans les Landes. Des différences significatives de sensibilité entre les 16 populations (provenances pures et hybrides) ont été mises en évidence. La variabilité entre populations a été scindée en 2 sources différentes : type de population (provenances pures ou hybrides) et provenance. Les hybrides sont significativement plus vigoureux, plus précoces et plus sensibles que les provenances pures correspondantes. Des différences significatives existent également entre provenances pour la vigueur, la précocité et la sensibilité. Une liaison positive a été observée entre sensibilité et vigueur des pins, et surtout entre sensibilité et précocité. Cela conduit à des effets et des classements différents pour les caractères de sensibilité ajustés ou non à la vigueur et à la précocité. Ainsi, la provenance Tamjout (Maroc) apparaît la plus précoce et la plus infectée, bien qu'elle ne présente pas la sensibilité intrinsèque la plus forte. L'augmentation de sensibilité avec la précocité s'explique par une meilleure coïncidence phénologique entre hôte et parasite (suivie dans le dispositif) qui accroît la probabilité d'infection. La variabilité interraciale ainsi mise en évidence ne permet pas dans l'immédiat de créer une variété de pin maritime résistante à la rouille, aucune race ou hybride n'apparaissant en moyenne plus résistant que la race landaise.
\end{abstract}

\section{Melampsora pinitorqua / rouille / pin maritime / provenance / phénologie}

Summary - Variation in susceptibility to twisting rust between maritime pine races. The susceptibility to twisting rust (Melampsora pinitorqua) of 16 maritime pine provenances (from different geographic races) and inter-provenance hybrids (between the Landes provenance and another geographic race) was studied under natural conditions in the Landes area. Growth traits (vigour and precocity) were also recorded. The 16 populations (pure provenances and hybrids) evidenced significantly different susceptibility, vigour and precocity traits (table $\mathrm{IV}$ ). The population effect resulted both from a provenance effect and a "population type" (pure provenance or hybrid) effect (table V). Provenance effects could be adequately described on a multivariate basis by discriminant analysis. The first axis (57.4\% discrimination) principally separated the Tamjout provenance (Morocco), which was very susceptible and precocious, from other provenances. The Italian and southeastern French provenances on the one hand, the lberian provenances on the other, were separated on the second axis (18.1\% discrimination), principally correlated to vigour (fig 3 , table VII). Hybrids proved to be sig- 
nificantly more vigorous, precocious and susceptible than the corresponding pure provenances. Positive correlations were observed between susceptibility to rust and pine vigour, and even more between susceptibility and precocity. This led to different effects and rankings of susceptibility variables when adjusted or not to vigour and phenology (tables VIII, IX). This was best exemplified by the Tamjout provenance, with the earliest growth and highest infection rate but a medium inherent susceptibility (tables VI, X). The dependence of susceptibility on precocity is explained by a better coincidence between host and parasite biological cycles (followed in the experimental design), leading to higher infection probability. Available results are not promising regarding the use of inter-racial variability for creating a maritime pine variety resistant to twisting rust. Indeed, no race or hybrid proved to be more resistant, on average, than the local race.

Melampsora pinitorqua / rust / maritime pine / provenance / phenology

\section{INTRODUCTION}

La rouille courbeuse des pins, causée par Melampsora pinitorqua Rostr, est décrite dans la plupart des pays d'Europe, sur pin sylvestre (Pinus sylvestris L) et pin maritime (Pinus pinaster Ait) principalement. Contrairement à d'autres espèces de $M e$ lampsora, l'existence de plusieurs races ou pathotypes de $M$ pinitorqua n'est pas connue. Les infections se produisent au printemps sur les pousses en élongation, uniquement sur les arbres jeunes, âgés de moins de 5 ans en général. Les dégâts se caractérisent par des dessèchements et déformations de pousses conduisant à des pertes en hauteur et surtout en qualité de la tige, lorsque la flèche est atteinte. En France, la maladie est endémique dans les Landes sur pin maritime. L'évolution actuelle de la sylviculture de cette essence, caractérisée par une intensification des pratiques sylvicoles, peut faire craindre pour le futur un impact accru de la maladie (Desprez-Loustau, 1990), comme cela a été observé dans des circonstances comparables pour la rouille fusiforme des pins du Sud-Est des ÉtatsUnis, Cronartium quercuum $\mathrm{f} s \mathrm{sp}$ fusiforme (Powers et al, 1981). Dans ce contexte, l'amélioration génétique de la résistance du pin maritime à la rouille courbeuse serait d'autant plus souhaitable.
L'espèce Pinus pinaster possède une aire naturelle morcelée, avec des caractéristiques écologiques très variées, qui a conduit à une diversification en races géographiques. La variabilité entre ces races a été décrite pour plusieurs caractères : croissance (Kremer et Roussel, 1986), résistance à la sécheresse (Guyon, 1980) ou aux insectes : Dioryctria splendidella (Sueron, 1982) ou Matsucoccus feytaudi même en l'absence de pression de sélection directe pour la résistance - (Ughetto, 1981). Par ailleurs, la variabilité a été appréhendée à l'aide de marqueurs contrôlés par des gènes neutres : terpènes (Baradat et Marpeau-Bezard, 1988) ou isozymes (Petit, 1988). L'hybridation de la race landaise avec d'autres races est une des voies envisagées pour l'amélioration génétique du pin maritime avec pour objectifs : la recherche et la fixation de gènes de résistance (à la sécheresse, aux maladies ou aux insectes), la recherche d'une complémentation favorable des caractéristiques de chaque race, ou éventuellement l'exploitation d'un effet d'hétérosis (Baradat et al, 1990). L'objectif de cette étude est de déterminer les possibilités ouvertes par la variabilité géographique du pin maritime et la création d'hybrides interraciaux pour l'amélioration de la résistance à la rouille courbeuse. 


\section{MATÉRIEL ET MÉTHODES}

\section{Matériel}

\section{Matériel végétal}

Seize populations élémentaires ont été comparées : 9 provenances pures, appartenant en général à des races géographiques très différentes, et des hybrides moyens correspondant au croisement de 7 d'entre elles avec la race landaise.

Le matériei représentant les races géographiques a été constitué par des graines récoltées dans les peuplements de l'aire naturelle avec, en complément, des graines issues de croisements intraraciaux. Les principales caractéristiques des zones géographiques sont résumées dans le tableau I. $M$ pinitorqua n'a été mentionné que dans les Landes et en Italie.

Les hybrides ont été constitués par la pollinisation de 5 arbres mères d'une provenance par un mélange du pollen de 10 arbres d'une autre provenance. Des croisements réciproques ont été utilisés en permutant mères et pères pour réaliser les mêmes hybrides. Les familles correspondant à la même combinaison de provenances ont été confondues lors de la réalisation du dispositif. Les effectifs varient entre 9 et 82 arbres par population élémentaire et entre 30 et 115 globalement pour les 7 provenances utilisées à la fois en provenance pure ou en hybridation avec la race landaise.

L'ensemble des pins ont été plantés à l'automne 1980 , après semis en pépinière pendant l'été de la même année.

\section{Inoculum}

L'inoculum naturel de rouille a été renforcé de la façon suivante. Des trembles (hôtes alternants de la rouille) appartenant au clone 117-50-1 connu pour sa sensibilité au $M$ pinitorqua (Desprez, 1980) ont été interplantés régulièrement dans le dispositif, à raison de 1 tremble pour 18 pins. Les trembles ont été inoculés artificiellement durant l'été 1982 par pulvérisation d'une suspension d'éciospores, récoltées sur pousses infectees de pins maritimes (hors du dispositif). La concentration était d'environ 20000 écio- spores/ml d'eau faiblement gélosée. Les plants inoculés ont été maintenus ensachés pendant une nuit.

\section{Méthodes}

\section{Dispositif expérimental}

La parcelle où est implanté le dispositif est située à Pierroton (Gironde) en lande mésophile. II s'agit d'un dispositif en blocs incomplets à composition aléatoire, les 16 provenances et hybrides étant répartis dans 26 blocs avec des parcelles unitaires de 5 plants ( 6 parcelles unitaires par bloc). Le nombre de répétitions par population élémentaire varie de 5 à 22 .

\section{Observations}

Elles ont effectuées le 6 juin 1983, ainsi que les $4,14,25$ mai et 6 juin 1984. La première année, elles ont porté sur les infections et la croissance des pins. La seconde année, elles ont concerné également la phénologie des pins et de la rouille.

\section{Infections}

Les observations suivantes ont été effectuées (les variables de sensibilité sont notées "S":

- nombre de chancres sur la pousse apicale : $S A_{83}, S A_{84}$ (6 juin);

- nombre de pousses latérales infectées $\left(N L I_{i}\right)$ et nombre total de pousses latérales (NLT $)$ sur le dernier verticille.

La variable $S P_{i}\left(S P_{83}, S P_{84}\right)$, pourcentage de pousses terminales infectées, a été calculée de la façon suivante :

$$
\begin{gathered}
S P_{i}=100 \times N L_{i} /\left(N L T_{i}+1\right), \text { si } S A_{i}=0 \\
S P_{i}=100 \times\left(N L I_{i}+1\right) /\left(N L T_{i}+1\right), \text { si } S A_{i}>0
\end{gathered}
$$

\section{Caractères de croissance} et de phénologie des pins

Les caractères observés sont les suivants :

- la hauteur des plants fin 1982: $\mathrm{H}_{82}$;

- la longueur de la pousse apicale : $L_{83}$ (6 juin), $L_{84-1}$ (4 mai), $L_{84-2}$ (14 mai), $L_{84-3}$ (25 mai), $L_{84}$ 
(6 juin); les accroissements relatifs en pourcentage de la longueur de la pousse 1984 en ont été déduits : $\Delta L_{1}=100 \times L_{84-1} / L_{84}, \Delta L_{2}=100 \times$ $\left(L_{84-2}-L_{84-1}\right) / L_{84}, \Delta L_{3}=100 \times\left(L_{84-3}-L_{84-2}\right)$ $/ L_{84}$

- le stade phénologique de la pousse apicale : $\mathrm{PH}_{1}$ (4 mai 1984), $P H_{2}$ (14 mai 1984), $\mathrm{PH}_{3}$ (25 mai 1984).

Les stades phénologiques sont ceux décrits par Debazac (1966) :

$-B_{0}$ : bourgeon dormant;

$-B_{1}$ : bourgeon en début d'élongation, avec écailles jointives;

- $B_{2}$ : écailles découvrant la pousse;

$-B_{3}$ : dégagement des brachyblastes;

$-B_{4}$ : apparition des pseudophylles;

$-B_{5}$ : décollement des 2 pseudophylles d'un même fascicule.

À chaque stade a été attribuée la note correspondante allant de 0 à 5 .

\section{Phénologie de la rouille}

La capacité germinative des téliospores, c'est-àdire leur aptitude à émettre des basidiospores infectieuses pour les pins, a été étudiée en conditions contrôlées sur des échantillons de rouille récoltés à proximité du dispositif aux différentes dates. Chaque échantillon était constitué de 10 feuilles de tremble abondamment infectées par la rouille. Celles-ci ont été mises à tremper au laboratoire pendant $2 \mathrm{~h}$ dans de l'eau de conduite. Un disque de $6 \mathrm{~mm}$ de diamètre a ensuite été découpé dans chacune des 10 feuilles. Les 10 disques ont été placés sur un papier filtre humide à l'intérieur du couvercle d'une boîte de Petri, placée à $17^{\circ} \mathrm{C}$. Après $24 \mathrm{~h}$, une observation au microscope a été effectuée sur la quantité de basidiospores obtenues dans le fond des boîtes dans chaque "aire de projection" en regard d'un disque; l'abondance de basidiospores a été estimée par une note allant de o à 4 (émission nulle à très forte). On en a dé-

Tableau I. Principales caractéristiques écologiques des stations de récolte des graines.

\begin{tabular}{|c|c|c|c|c|}
\hline Station & Situation & Altitude & Climat & Sol \\
\hline Tabarka & $\begin{array}{l}\text { Tunisie } \\
\text { (Ouest) }\end{array}$ & $<570 \mathrm{~m}$ & $\begin{array}{l}\text { Humide, } \\
\text { à hiver chaud }\end{array}$ & $\begin{array}{l}\text { Sol brun superficiel } \\
\text { sur grès }\end{array}$ \\
\hline Tamjout & $\begin{array}{l}\text { Maroc } \\
\text { (Est) }\end{array}$ & $\begin{array}{l}1650 \mathrm{~m} \\
\text { Moyen Atlas }\end{array}$ & $\begin{array}{l}\text { Mméditérranéen } \\
\text { semi-aride }\end{array}$ & $\begin{array}{l}\text { Sol brun superficiel } \\
\text { sur grès calcaire }\end{array}$ \\
\hline Leiria & $\begin{array}{l}\text { Portugal } \\
\text { (Ouest) }\end{array}$ & $\begin{array}{l}<200 \mathrm{~m} \\
\text { (côte) }\end{array}$ & Océanique & Vieille dune \\
\hline Cazorla & Espagne & $\begin{array}{l}1000 \mathrm{~m} \\
\text { Sierra Nevada }\end{array}$ & Semi-aride & Dolomitique \\
\hline Gênes & Italie & $300 \mathrm{~m}$ & Méditerranéen & $\begin{array}{l}\text { Sable océanique } \\
\text { sur schistes }\end{array}$ \\
\hline Landes & France & $10-20 \mathrm{~m}$ & Océanique & $\begin{array}{l}\text { Podzol } \\
\text { sableux }\end{array}$ \\
\hline Esterel & France & $410 \mathrm{~m}$ & $\begin{array}{l}\text { Méditerranéen } \\
\text { humide }\end{array}$ & $\begin{array}{l}\text { Sol brun superficiel } \\
\text { sur grès }\end{array}$ \\
\hline Porto-Vecchio & Corse & $50-200 \mathrm{~m}$ & $\begin{array}{l}\text { Méditerranéen } \\
\text { humide }\end{array}$ & $\begin{array}{l}\text { Sol brun superficiel } \\
\text { sur roche-mère siliceuse }\end{array}$ \\
\hline Vivario & Corse & $600 \mathrm{~m}$ & $\begin{array}{l}\text { Méditerranéen } \\
\text { humide }\end{array}$ & $\begin{array}{l}\text { Sol brun superficiel } \\
\text { sur roche-mère siliceuse }\end{array}$ \\
\hline
\end{tabular}


duit la note moyenne d'émission de basidiospores à chaque date.

\section{Analyse du dispositif expérimental}

Le traitement biométrique a été effectué en utilisant la bibliothèque de programmes OPEP ( $\mathrm{Ba}$ radat, 1989).

\section{Analyse factorielle des correspondances}

Une analyse factorielle des correspondances a été effectuée à partir des notes de phénologie de 1984 aux 3 dates $\left(P H_{1}, P H_{2}, P H_{3}\right)$.

On a procédé, pour chaque individu, à un recodage disjonctif complet de la note moyenne sur les 3 dates: $P H=\left(P H_{1}+P H_{2}+P H_{3}\right) / 3$. La valeur entière la plus proche de cette moyenne : $B_{i}=I N T(P H+0,5)$ génère la variable qualitative $V_{i}=0$ ou 1 suivant qu'elle n'est pas ou qu'elle est réalisée. $B_{i}$ n'est autre que le stade phénologique le plus proche de la note moyenne. Le codage obtenu est représenté tableau II pour les 4 valeurs possibles de $B_{i}(1,2$, 3, ou 4).

L'AFC a été faite sur le tableau de contingence croisant les 4 variables qualitatives avec les 16 provenances ou hybrides (dénombrement des individus possédant la valeur 1 pour chaque population et chaque variable).

\section{Analyses de variance}

Des analyses de variances multivariables ont été effectuées sur les 9 variables $H_{82}, L_{83}, L_{84}$, $\log \left(\Delta L_{1}+1\right)$ [notée par la suite $\Delta L_{1}{ }^{*}$ ] $, \Delta L_{2}, \Delta L_{3}$, $\log \left(S P_{83}+1\right)\left[S P_{83}{ }^{*}\right], \log \left(S P_{84}+1\right)\left[S P_{84}{ }^{*}\right], P H$, selon un modèle non orthogonal à effets fixes (Searle, 1971). La variable SA n'a pas été analysée en tant que telle. Seules ont été conservées pour les analyses les parcelles unitaires comportant au moins 2 individus notés pour l'ensemble des caractères.

Une première analyse en classification croisée a été effectuée sur les 26 niveaux du facteur bloc et les 16 niveaux du facteur population (provenance ou hybride), selon le modèle :

$$
Y_{i j k}=\mu+p_{i}+b_{j}+(p b)_{i j}+e_{i j k}
$$

$p_{i}$ : effet de la population $i$

$b_{j}:$ effet du bloc $j$

$(p b)_{i j}$ : interaction entre la population $i$ et le bloc $j$ $e_{i j k}$ : effet individuel intraparcelle unitaire.

Compte tenu de l'écart important à la normalité de la distribution des termes d'erreurs individuels $\left(e_{i j k}\right)$ pour la plupart des caractères, les tests $F$ ont été réalisés par rapport aux carrés moyens d'interaction, c'est-à-dire en tronquant le modèle au niveau des parcelles unitaires. On sait (Philoche, 1977) que seule la symétrie de la distribution des résidus intervient dans la loi de distribution du test $F$. Nous avons donc testé ce paramètre en utilisant le test de symétrie de Pearson (Snedecor et Cochran, 1967). L'hypothèse de symétrie n'a été rejetée que dans un cas $\left(\Delta L_{3}\right)$ et seulement au seuil $5 \%$ (pas au seuil $1 \%)$.

Selon le modèle précédemment décrit, un fichier de données individuelles ajustées aux effets blocs, $Y^{\prime}{ }_{i j k}$ a été constitué :

$$
Y_{i j k}^{\prime}=Y_{i j k}-\widehat{b_{j}}
$$

en ne conservant que le sous-ensemble des 7 provenances représentées à la fois en provenance pure et en hybridation avec la race landaise (élimination des provenances Landes et Porto-Vecchio).

Tableau II. Codage disjonctif complet des notes de phénologie.

\begin{tabular}{lccccc}
$\begin{array}{l}\text { Plage de variation } \\
\text { de la moyenne }\end{array}$ & $\begin{array}{c}\text { Stade phénologique } \\
\text { le plus proche }\end{array}$ & \multicolumn{3}{c}{ Variables qualitatives } \\
& & $V_{1}$ & $V_{2}$ & $V_{3}$ & $V_{4}$ \\
\hline $0,5 \leq \mathrm{PH}<1,5$ & $\mathrm{~B}_{1}$ & 1 & 0 & 0 & 0 \\
$1,5 \leq \mathrm{PH}<2,5$ & $\mathrm{~B}_{2}$ & 0 & 1 & 0 & 0 \\
$2,5 \leq \mathrm{PH}<3,5$ & $\mathrm{~B}_{3}$ & 0 & 0 & 1 & 0 \\
$3,5 \leq \mathrm{PH}<4,5$ & $\mathrm{~B}_{4}$ & 0 & 0 & 0 & 1 \\
& & & & & \\
\hline
\end{tabular}


Sur ce nouveau fichier, une deuxième analyse de variance a été faite en traitant comme valeurs élémentaires les moyennes de parcelles unitaires ajustées à l'effet bloc. Le modèle global est donc à 3 facteurs en classification croisée : bloc, type de population (2 niveaux : provenance pure et hybride) et provenance (7 niveaux). II s'écrit :

$$
Y_{j l m}=\mu+b_{j}+t_{1}+g_{m}+(t g)_{l m}+e_{j l m}
$$

$b_{j}=$ effet du bloc $j$

$t_{l}=$ effet du type de population $/$

$g_{m}=$ effet de la provenance (origine géographique) $m$

$(\mathrm{tg})_{I m}=$ interaction entre le type de population I et la provenance $m$

$e_{j l m}=$ erreur résiduelle.

Dans cette deuxième analyse de variance, le carré moyen d'erreur utilisé a été le carré moyen intrapopulation correspondant aux termes $e_{j m}$. La symétrie de la distribution de ces nouveaux résidus a été testée comme précédemment. Un seul cas de dissymétrie significative, au seuil $5 \%$ seulement, a été observé $\left(S P_{84}{ }^{*}\right)$, l'examen de l'histogramme de la distribution montrant que cette dissymétrie était peu accentuée.

L'hypothèse d'homoscédasticité entre les 14 combinaisons provenance $\times$ type de population n'a été rejetée, au seuil de $5 \%$ seulement, que pour 2 caractères $\left(\Delta L_{1}{ }^{*}\right.$ et $\left.\Delta L_{2}\right)$.

Les effets population, type de population et provenance, ajustés à l'effet bloc, ont été comparés deux à deux par des tests $t$, en raison des disparités d'effectifs. Chaque comparaison a été réalisée au seuil de $5 \%$, la méthode de Bonferroni du «test $t$ corrigé" étant jugée trop sévère.

\section{Analyse discriminante}

Une analyse discriminante a été effectuée selon le modèle de la deuxième analyse de variance sur les 7 niveaux du facteur provenance. Les variables prises en compte sont celles de l'analyse de variance. Le test de Kullback (Legendre et Legendre, 1984) n'a pas montré d'hétérogénéité des matrices de variances-covariances entre les 14 combinaisons provenance $x$ type de population : $\chi^{2}=96,46,135 \mathrm{dl}(\rho: 0,99)$.

\section{Analyse de covariance}

Nous avons cherché à comparer la sensibilité entre populations mesurée en 1983 et 1984
$\left(S P_{83}{ }^{*}\right.$ et $\left.S P_{84}{ }^{*}\right)$ en éliminant l'effet des différences d'élongation des pousses $\left(L_{83}\right.$ et $\left.L_{84}\right)$ et de précocité phénologique $(P H)$. Cette étude concerne les effets principaux définis dans la deuxième analyse : effet provenance et effet type de population.

L'importance des 3 covariables $\left(P H, L_{83}\right.$ et $\left.L_{84}\right)$ pour la prédiction des 2 variables expliquées $\left(S P_{84}{ }^{*}\right.$ et $\left.S P_{83}{ }^{*}\right)$ a été étudiée par la méthode descendante (Snedecor et Cochran, 1967). Les comparaisons des effets provenance et type de population ont alors été effectuées, i) après ajustement à la covariable la plus importante, ii) après ajustement aux covariables significatives.

\section{RÉSULTATS}

\section{Observations générales sur l'évolution du parasite, de l'hôte et des infections (1984)}

L'évolution de la capacité germinative des téliospores et de la phénologie des pins en 1984 est schématisée par la figure 1. On observe une diminution progressive de l'émission potentielle des basidiospores au cours du mois de mai, celle-ci étant très faible ou nulle le 25. La majorité des pins présente respectivement les stades $B_{1}, B_{3}$ et $\mathrm{B}_{4}$ aux 3 dates 4,14 et 25 mai.

Les premiers symptômes de rouille sont apparus entre le 14 et le 25 mai. Le nombre d'infections a augmenté sensiblement entre le 25 mai et le 6 juin, passant de 19 à $35 \%$ de pins infectés sur l'ensemble du dispositif.

\section{Comparaison des populations}

\section{Analyse factorielle des correspondances (phénologie)}

L'analyse effectuée sur la notion de phénologie permet une bonne représentation des 
phénologie pins

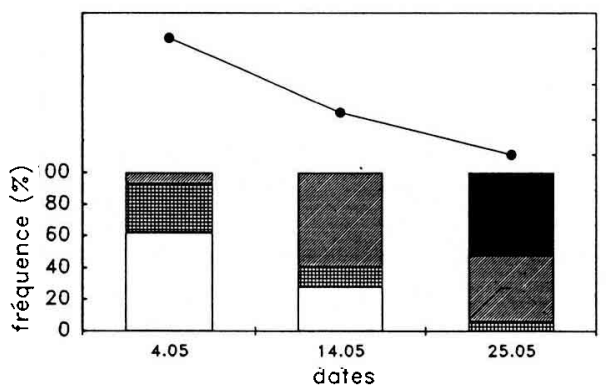

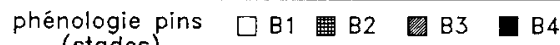
(stades)

phénologie rouille

(potentiel d'émission de basidiospores)

Fig 1. Évolution phénologique moyenne des pins (toutes provenances confondues) et de la rouille au cours du printemps 1984 (stade $B_{2}=$ début de la période de sensibilité des pins à la rouille).

différentes populations sur le plan défini par les 2 premiers axes (fig 2). L'axe 1, qui correspond à $70,0 \%$ de l'inertie totale, est expliqué principalement par la variable associée à la présence du stade $B_{4}$ (présen-

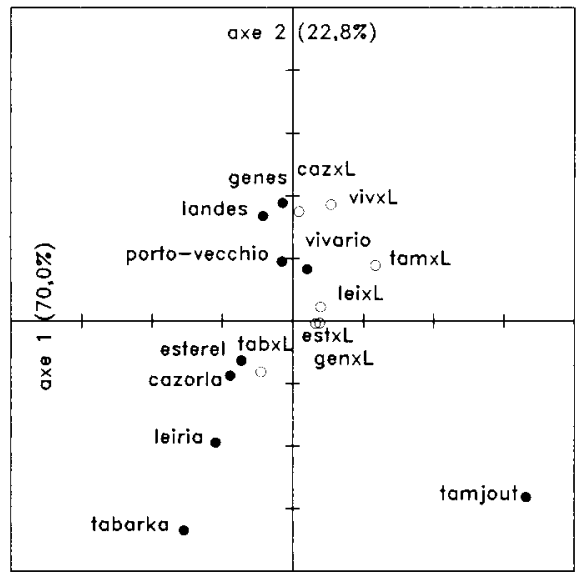

Fig 2. Analyse factorielle des correspondances sur la phénologie des populations en 1984. tant une contribution absolue de 0,66). La population Tamjout pure présente une contribution absolue très importante pour cet axe $(0,61)$. La plupart des populations hybrides, et presque elles seules (à l'exception de Tamjout) sont représentées dans le demi-plan défini par des coordonnées positives sur l'axe 1, correspondant à une plus grande précocité. L'axe $2(22,8 \%$ de l'inertie totale) oppose principalement la variable 3 (stade $B_{3}$, coordonnées positives, contribution de 0,46 ) aux variables 1 et 4 (stades $B_{1}$ et $B_{4}$, coordonnées négatives, contributions de 0,24 et 0,23 ).

\section{Étude des effets population (ensemble des caractères)}

L'analyse de variance permet de mettre en évidence un effet population très significatif pour tous les caractères de vigueur : hauteur et longueurs de la pousse apicale (tableau III). La phénologie et la précocité d'élongation de la pousse apicale (exprimée par $\left.\Delta L 1^{*}\right)$ varient aussi significativement. Concernant les caractères de sensibilité, seul celui noté en 1984 permet une distinction significative des populations. La sensibilité mesurée en 1984 est significativement corrélée avec celle mesurée en 1983 : corrélation de rangs $S P_{83}{ }^{*}-S P_{84}{ }^{*}$ (coefficient de Spearman) $=0,57,14 \mathrm{dl}$ $(P<5 \%)$. On observe d'autre part une liaison positive significative entre sensibilité et vigueur (corrélation de rangs $S P_{84}{ }^{*}-L_{84}$ et $S P_{84}{ }^{*}-L_{83}$ de 0,72 et 0,80 respectivement, probabilité $<1 \%$ ) et entre sensibilité et phénologie (corrélation de rangs $\mathrm{SP}_{84}{ }^{*}$ - PH : 0,73). Les 6 populations les plus sensibles figurent également parmi les plus précoces (caractère $P H$ ), à l'exception de Tabarka x Landes, et les plus vigoureuses, sauf Tamjout (tableau IV). La provenance landaise apparaît relativement tardive, peu vigoureuse et peu sensible. 
Tableau III. Analyse de variance bloc $x$ population : tests $F$ du facteur population ajusté.

\begin{tabular}{clllllllll}
\hline & $H_{82}$ & $L_{83}$ & $L_{84}$ & $\Delta L_{1}$ & $\Delta L_{2}$ & $\Delta L_{3}$ & $P H$ & $S P_{83}{ }^{*}$ & $S P_{84}{ }^{*}$ \\
\hline$F$ & 4,70 & 3,45 & 3,95 & 2,61 & 0,39 & 1,54 & 8,05 & 1,18 & 1,92 \\
$P(\%)$ & 0,0 & 0,0 & 0,0 & 0,2 & 97,9 & 10,7 & 0,0 & 30,3 & 3,0 \\
$(15,98 \mathrm{dl})$ & & & & & & & & & \\
\hline
\end{tabular}

$H$ : hauteur de l'arbre; $L$ : longueur de la pousse apicale; $\Delta L_{i}$ : accroissement relatif de la pousse apicale à la $P$ date de notation; $P H$ : note moyenne de phénologie; $S P$ : proportion de pousses terminales infectées; * transformation logarithmique.

\section{Étude des effets provenance et type de population}

\section{Analyse de variance}

L'analyse des données ajustées à l'effet bloc révèle un effet provenance et un effet type de population significatifs pour les ca- ractères de sensibilité $\left(S P_{84}{ }^{\star}\right)$, de vigueur $(H, L)$ et de phénologie $(P H)$ (tableau V). Les hybrides sont significativement plus vigoureux, plus précoces et plus sensibles que les provenances pures (tests $t$ sur les caractères $\mathrm{L}, \mathrm{PH}$ et $S P_{84}{ }^{*}$ ). Seul l'effet provenance est significatif pour la précocité

Tableau IV. Analyse de variance bloc $x$ population : comparaison des moyennes par population ajustées à l'effet bloc, par des tests $t$.

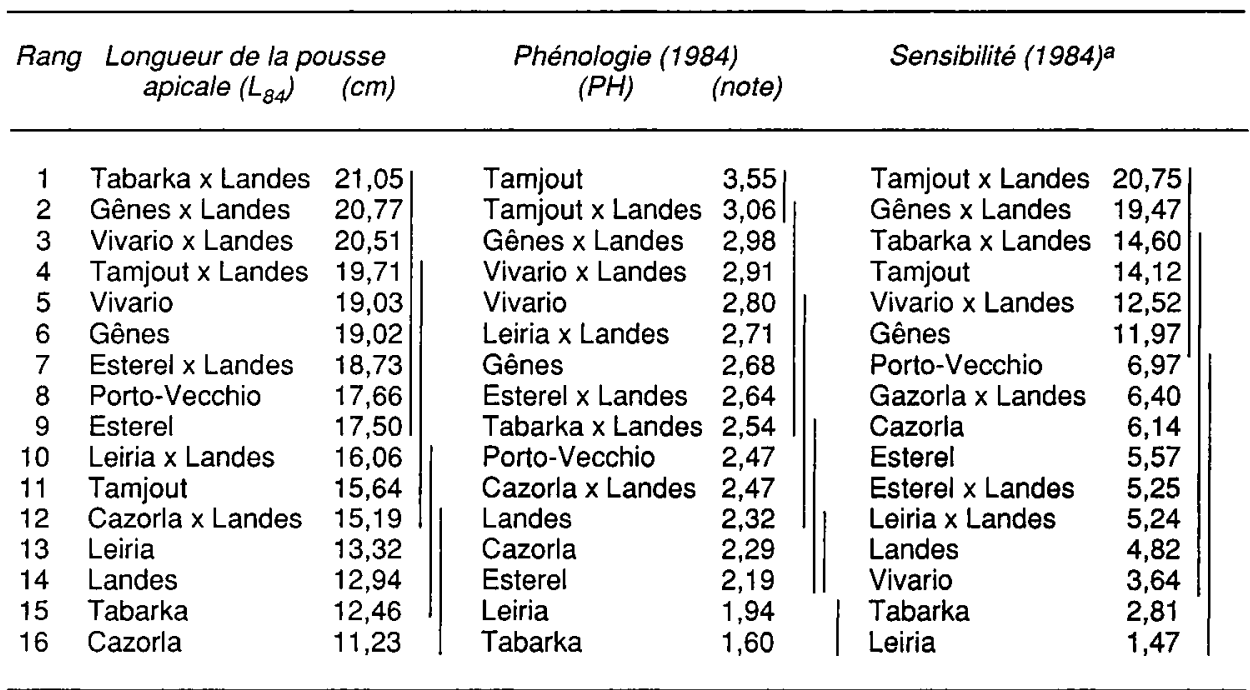

a : les valeurs données sont celles de la variable non transtormée (pourcentage de pousses attaquées), les tests ayant été faits sur la variable transformée : $\log \left(S P_{84}+1\right)$. Les modalités reliées par un trait ne diffèrent pas significativement au seuil de $5 \%$. 
Tableau V. Analyse de variance provenance $\mathrm{x}$ type de population : tests $F$ des facteurs ajustés et de l'interaction.

\begin{tabular}{|c|c|c|c|c|c|c|c|c|c|}
\hline & $H_{82}$ & $L_{83}$ & $L_{84}$ & $\Delta L_{1}{ }^{*}$ & $\Delta L_{2}$ & $\Delta L_{3}$ & $\mathrm{PH}$ & $S P_{83}{ }^{*}$ & $S P_{84}{ }^{*}$ \\
\hline \multicolumn{10}{|l|}{ Provenance } \\
\hline$F$ & 5,36 & 5,16 & 6,72 & 4,40 & 0,79 & 2,07 & 18,58 & 2,78 & 3,57 \\
\hline $\begin{array}{l}P(\%) \\
(6,84 \mathrm{dl})\end{array}$ & 0,0 & 0,0 & 0,0 & 0,1 & 58,0 & 6,4 & 0,0 & 1,6 & 0,3 \\
\hline \multicolumn{10}{|l|}{ Type de pop } \\
\hline$F$ & 31,67 & 12,26 & 10,22 & 0,53 & 0,02 & 3,28 & 25,51 & 1,37 & 9,56 \\
\hline $\begin{array}{l}P(\%) \\
(184 \mathrm{dl})\end{array}$ & 0,0 & 0,1 & 0,2 & 52,6 & 87,8 & 7,0 & 0,0 & 24,3 & 0,3 \\
\hline \multicolumn{10}{|l|}{$\begin{array}{l}\text { Interaction } \\
\text { prov } x \text { type }\end{array}$} \\
\hline$F$ & 0,59 & 1,12 & 1,03 & 0,78 & 0,39 & 2,23 & 3,47 & 1,13 & 0,57 \\
\hline$P(\%)$ & 73,6 & 35,9 & 41,2 & 59,2 & 88,4 & 4,8 & 0,4 & 35,0 & 75,7 \\
\hline
\end{tabular}

$H$ : hauteur de l'arbre; $L$ : longueur de la pousse apicale; $\Delta L i$ : accroissement relatif de la pousse apicale à la ie date de notation; $P H$ : note moyenne de phénologie; SP : proportion de pousses terminales infectées; * transformation logarithmique.

d'élongation $\left(\Delta L_{1}{ }^{*}\right)$ et le pourcentage de pousses infectées noté en 1983. Tamjout et Leiria sont respectivement la provenance la plus sensible et la plus résistante pour les 2 années (tableau VI). Tamjout est également significativement plus précoce que toutes les autres provenances. Enfin on observe une interaction provenance $x$ type de population pour le caractère de phénologie $(P H)$. L'essentiel de cette interaction est dû aux 2 provenances qui présentent précisément des caractéristiques phénologiques extrêmes: Tamjout et Tabarka, comme cela est exprimé par les écovalences relatives ${ }^{1}$ (Tamjout : 51,35 ; Tabarka : 25,92 , soit $77 \%$ du total cumulé à elles deux).

\section{Analyse discriminante de l'effet provenance}

Les 3 premiers axes permettent une discrimination significative des provenances, les 2 premiers axes expliquant $75 \%$ de la discrimination totale. L'axe 1 est très lié aux caractères de précocité et de sensibilité, l'axe 2 à ceux de vigueur, et de sensibilité en 1984 dans une moindre mesure (tableau VII). Les provenances Tamjout et Tabarka se distinguent de toutes les autres, aux 2 extrémités de l'axe 1. Deux groupes intermédiaires sont constitués d'une part des provenances ibériques, d'autre part des provenances italienne et françaises du Sud-Est (fig 3).

\footnotetext{
1 C'est-à-dire, le pourcentage de la somme totale des carrés d'interaction attribuable à chaque provenance (Baradat, 1989).
} 
Tableau VI. Analyse de variance provenance $x$ type de population : comparaison des moyennes par provenance ajustées à l'effet type de population, par des test $t$.

\begin{tabular}{|c|c|c|c|c|c|c|c|c|c|c|}
\hline \multirow{3}{*}{$\begin{array}{l}\text { Rang } \\
1\end{array}$} & \multirow{2}{*}{\multicolumn{4}{|c|}{$\begin{array}{l}\text { Longueur de la pousse apicale } \\
L_{83}(\mathrm{~cm}) \quad L_{84}(\mathrm{~cm})\end{array}$}} & \multirow{2}{*}{\multicolumn{2}{|c|}{$\begin{array}{l}\text { Phénologie } \\
\text { PH (note) }\end{array}$}} & \multicolumn{4}{|c|}{ Sensibilité } \\
\hline & & & & & & & $S P$ & & $S P_{8}$ & \\
\hline & Vivario & 17,87 & Gênes & 19,29 & Tamjout & 3,51 & Tamjout & 17,91 & Tamjout & 17,13 \\
\hline 2 & Gênes & 17,24 & Vivario & 19,27 & Vivario & 2,79 & Vivario & 10,78 & Gênes & 15,26 \\
\hline 3 & Esterel & 17,02 & Esterel & 18,05 & Gênes & 2,76 & Esterel & 9,58 & Tabarka & 9,04 \\
\hline 4 & Tamjout & 16,83 & Tabarka & 17,47 & Cazorla & 2,35 & Gênes & 8,22 & Vivario & 7,91 \\
\hline 5 & Cazorla & 13,96 & Tamjout & 17,16 & Esterel & 2,35 & Cazorla & 7,55 & Cazorla & 6,76 \\
\hline 6 & Tabarka & 13,52 & Leiria & 14,50 & Leiria & 2,29 & Tabarka & 6,85 & Esterel & 6,26 \\
\hline 7 & Leiria & 13,13 & Cazorla & 12,57 & Tabarka & 2,13 & Leiria & 3,86 & Lelria & 3,02 \\
\hline
\end{tabular}

a : les valeurs données sont celles de la variables non transformée (pourcentage de pousses altaquées), les tests ayant été faits sur la variable transformée : $\log (S P i+1)$. Les modalités relieeses par un trait ne dlfférent pas significativement au seuil de $5 \%$.

Analyse de covariance (comparaison de la sensibilité à vigueur et/ou précocité constante)

Pour les 2 variables de sensibilité, mesurées en $1983\left(S P_{83}{ }^{*}\right)$ et $1984\left(S P_{84}{ }^{*}\right)$, la meilleure variable prédictrice est celle de phénologie $(P H)$, suivie de celle de vigueur mesurée la même année $\left(L_{83}\right.$ et $L_{84}$ respectivement). Dans les 2 cas, l'apport de la variable de vigueur mesurée l'année sui-

Tableau VII. Analyse discriminante de l'effet provenance : caractéristiques des axes; corrélations entre variables canoniques et effets provenance pour les différents caractères.

\begin{tabular}{|c|c|c|c|c|c|c|c|c|c|}
\hline & $\begin{array}{l}\text { Numéro } \\
\text { de l'axe }\end{array}$ & \multicolumn{3}{|c|}{ Discrimination (\%) } & $\chi^{2}$ & $\begin{array}{l}\text { Degrés } \\
\text { de liberté }\end{array}$ & \multicolumn{3}{|c|}{$\begin{array}{r}P \\
(\%)\end{array}$} \\
\hline & $\begin{array}{l}1 \\
2 \\
3\end{array}$ & \multicolumn{2}{|c|}{$\begin{array}{l}57,35 \\
18,09 \\
16,85\end{array}$} & \multicolumn{2}{|c|}{$\begin{array}{l}84,81 \\
38,09 \\
36,03\end{array}$} & $\begin{array}{l}14 \\
12 \\
10\end{array}$ & \multicolumn{3}{|c|}{$\begin{array}{l}0,00 \\
0,02 \\
0,01\end{array}$} \\
\hline & $H_{82}$ & $L_{83}$ & $L_{84}$ & $\Delta L_{1}^{*}$ & $\Delta L_{2}$ & $\Delta L_{3}$ & $\mathrm{PH}$ & $S P_{83}$ & $S P_{84}$ \\
\hline $\begin{array}{l}V C 1 \\
V C 2 \\
V C 3\end{array}$ & $\begin{array}{r}0,31 \\
-0,91 \\
-0,02\end{array}$ & $\begin{array}{r}-0,30 \\
-0,87 \\
0,23\end{array}$ & $\begin{array}{r}-0,14 \\
-0,69 \\
0,66\end{array}$ & $\begin{array}{r}-0,79 \\
-0,02 \\
0,53\end{array}$ & $\begin{array}{r}-0,85 \\
0,26 \\
0,28\end{array}$ & $\begin{array}{r}0,72 \\
-0,34 \\
0,17\end{array}$ & $\begin{array}{r}-0,94 \\
-0,32 \\
0,03\end{array}$ & $\begin{array}{r}-0,84 \\
-0,14 \\
0,05\end{array}$ & $\begin{array}{l}-0,61 \\
-0,50 \\
-0,16\end{array}$ \\
\hline
\end{tabular}

$H$ : hauteur de l'arbre; $L:$ longueur de la pousse apicale; $\Delta L_{i}$ : accroissement relatif de la pousse apicale à la ${ }^{\theta}$ date de notation; $P H$ : note moyenne de phénologie; $S P$ : proportion de pousses terminales infectées; * transformation logarithmique. 


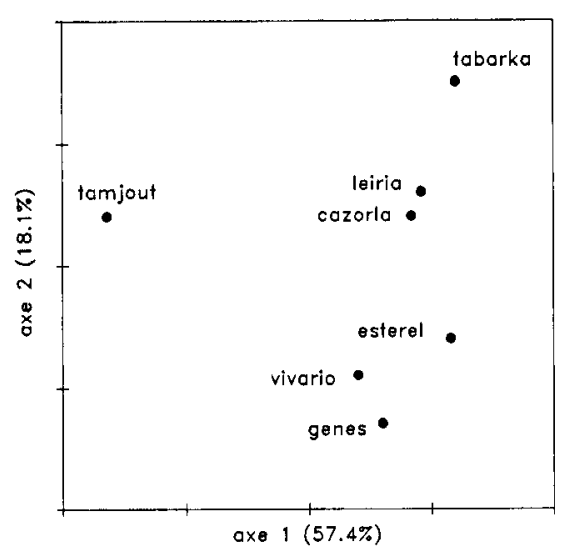

Fig 3. Analyse discriminante de l'effet provenance : représentation des centroïdes sur le plan défini par les 2 premiers axes. vante ou précédente ( $L_{84}$ et $L_{83}$ respectivement) n'améliore pas la précision de la prédiction (tableaux VIII et IX).

L'ajustement de $S P_{83}{ }^{*}$ et $S P_{84}{ }^{*}$ à la meilleure variable explicative ou aux 2 covariables significatives conduit à une modification des effets par rapport aux variables non ajustées (tableaux VIII et IX). L'effet type de population, préalablement non significatif pour $S P_{83}{ }^{*}$, perd également sa significativité pour $S P_{84}$ * dès l'ajustement à $P H$. De même, l'effet provenance, maintenu pour $S P_{84}{ }^{*}$, devient non significatif pour $S P_{83}{ }^{*}$, dès l'ajustement à $P H$.

Le classement des provenances pour la sensibilité mesurée en 1984 ajustée à la phénologie et à la vigueur apparaît très différent de celui précédemment obtenu sans ajustement (tableaux $\mathrm{VI}$ et $\mathrm{X}$ ). On peut noter en particulier le rang moyen de la provenance Tamjout.

Tableau VIII. Analyse de covariance sensibilité 1983 - phénologie, vigueur $\left(S P_{83}{ }^{*}-P H, L_{83}\right.$ et $\left.L_{84}\right)$ pour les effets provenance $x$ type de population.

\begin{tabular}{|c|c|c|c|c|}
\hline & Palier & $d l$ & $F$ & Probabilité (\%) \\
\hline \multicolumn{5}{|l|}{ Méthode descendante } \\
\hline Apport total erreur & 1 & 3 & 8,99 & 0,00 \\
\hline Apport total erreur & 2 & 2 & 13,49 & 0,00 \\
\hline$L_{84}$ & & 1 & 0,00 & 97,67 \\
\hline Apport total erreur & 3 & 1 & 17,92 & 0,01 \\
\hline$L_{83}$ & & 1 & 9,07 & 0,36 \\
\hline Effet type de pop ajusté & & 1 & 0,71 & 59,48 \\
\hline Effet provenance ajusté & & 6 & 1,07 & 39,01 \\
\hline Effet interaction ajusté & & 6 & 2,53 & 2,66 \\
\hline \multicolumn{5}{|c|}{ Modèle à 2 covariables ( $\mathrm{PH}$ et $\mathrm{L}_{83}$ ) } \\
\hline Apport total erreur & 1 & 2 & 13,66 & 0,00 \\
\hline Effet type de pop ajusté & & 1 & 2,31 & 12,84 \\
\hline Effet provenance ajusté & & 6 & 1,37 & 27,75 \\
\hline Effet interaction ajusté & & 6 & 2,39 & 3,49 \\
\hline
\end{tabular}

$S P$ : pourcentage de pousses terminales attaquées; $P H$ : note moyenne de phénologie; $L$ : longueur de la pousse apicale; " transformation logarithmique. 
Tableau IX Analyse de covariance sensibilité 1984 - phénologie, vigueur $\left(S P_{84}-P H, L_{83}\right.$ et $\left.L_{84}\right)$ pour les effets provenance $x$ type de population.

\begin{tabular}{|c|c|c|c|c|}
\hline & Palier & $d l$ & $F$ & Probabilité (\%) \\
\hline \multicolumn{5}{|l|}{ Méthode descendante } \\
\hline Apport total erreur & 1 & 3 & 12,54 & 0,00 \\
\hline Apport total erreur & 2 & 2 & 18,71 & 0,00 \\
\hline$L_{83}$ & & 1 & 0,21 & 65,26 \\
\hline Apport total erreur & 3 & 1 & 30,34 & 0,00 \\
\hline $\mathrm{L}_{84}$ & & 1 & 7,09 & 0,91 \\
\hline Effet type de pop ajusté & & 1 & 0,30 & 59,49 \\
\hline Effet provenance ajusté & & 6 & 2,21 & 5,00 \\
\hline Effet interaction ajusté & & 6 & 2,06 & 6,68 \\
\hline \multicolumn{5}{|c|}{ Modèle à 2 covariables ( $\left(\mathrm{HH}\right.$ et $\mathrm{L}_{84}$ ) } \\
\hline Apport total erreur & 1 & 2 & 18,89 & 0,00 \\
\hline Effet type de pop ajusté & & 1 & 0,20 & 65,99 \\
\hline Effet de provenance ajusté & & 6 & 2,24 & 4,74 \\
\hline Effet interaction ajusté & & 6 & 1,28 & 27,62 \\
\hline
\end{tabular}

SP : pourcentage de pousses terminales attaquées; $P H$ : notre moyenne de phénologie; $L:$ longueur de la pousse apicale; " transformation logarithmique.

\section{DISCUSSION}

Notre étude confirme la variabilité géographique du pin maritime pour tous les caractères étudiés : vigueur, précocité, sensibilité à la rouille. La vigueur relative des différentes provenances dans notre essai apparait assez différente de celle observée dans la plupart des études antérieures, notamment pour les performances relativement mauvaises des provenances atlantiques, Landes et Leiria. Cela pourrait s'expliquer en partie par l'âge des arbres. En effet, nos observations ont eu lieu au cours des $4^{\mathrm{e}}$ et $5^{\mathrm{e}}$ saisons de végétation des pins (correspondant à l'âge moyen de sensibilité à la rouilie), alors que la pleine expression du génotype pour la hauteur n'est en général acquise qu'à partir de 810 ans (Baradat, 1989). Les arbres, dans
Tableau $X$. Analyse de covariance sensibilité 1984 - phénologie, vigueur $1984\left(S_{84}-(P H\right.$, $L_{84}$ ) : comparaison des effets provenance pour la sensibilité ajustée, par des tests $t$.

\section{Rang Provenance $S P_{84}$ ajustéa / $\left(P H, L_{84}\right)$}

\begin{tabular}{llrl}
\hline & & & \\
1 & Tabarka & 14,32 & abc \\
2 & Gênes & 12,96 & ab \\
3 & Cazorla & 8,56 & $\mathrm{a}$ \\
4 & Esterel & 8,31 & $\mathrm{abc}$ \\
5 & Tamjout & 7,41 & $\mathrm{abc}$ \\
6 & Vivario & 5,57 & $\mathrm{c}$ \\
7 & Leiria & 5,31 & $\mathrm{bc}$ \\
\hline
\end{tabular}

a : les valeurs données sont celles de la variable non transformée (pourcentage de pousses attaquées), les tests ayant été faits sur la variable transformée : log $\left(S P_{84}+1\right)$. Les modalités affectées d'une même lettre ne diffèrent pas significativement au seuil de $5 \%$. 
notre essai, manifestent encore probablement des effets maternels, qui affectent notamment la hauteur. Ces effets maternels pourraient être liés en particulier au poids des graines, comme cela a déjà été mentionné pour Leiria, à faible poids et faible vigueur initiale (Sarrauste, 1982). La précocité de croissance apparaît comme un caractère très discriminant des provenances. La grande précocité de Tamjout, dans les conditions landaises, est confirmée, en opposition à Tabarka, les autres provenances étant intermédiaires (Guyon, 1982; Kremer et Roussel, 1986). Enfin, notre étude montre que les différentes provenances se distinguent également par leur sensibilité à la rouille courbeuse. Cette sensibilité apparaît liée à la vigueur et surtout, à la précocité. L'ajustement des caractères de sensibilité à la phénologie et à la vigueur conduit à une perte de significativité de l'effet provenance en 1983 et à un classement différent des provenances en 1984. La sensibilité observée en conditions naturelles est donc sous forte dépendance de la croissance des pousses et ne reflète que partiellement la sensibilité intrinsèque des provenances, telle qu'on pourrait l'estimer en conditions contrôlées. La relation entre sensibilité et précocité s'explique sans doute par le phénomène biologique de coïncidence phénologique entre hôte et parasite. En effet, le suivi de la maturation des téliospores - c'est-à-dire leur aptitude à émettre des basidiospores infectieuses pour les pins - montre que l'optimum est atteint avant la date moyenne de début de sensibilité des pousses de pins (stade $B_{2}$ ). Une plus grande précocité se traduit donc par une exposition des pousses à une pression d'inoculum plus importante, d'où des risques d'infection accrus. Ainsi, la provenance Tamjout, très précoce, est celle qui présente le plus d'infections, bien qu'elle ne présente pas la sensibilité intrinsèque la plus forte (après ajustement à la phéno- logie et la vigueur). L'importance de la coincidence phénologique pour le risque d'infection par la rouille courbeuse a également été soulignée pour le pin noir et le pin sylvestre, notamment dans le cas d'essais comparatifs de provenances pour cette dernière espèce (Kurkela, 1974; Longo et al, 1980).

L'hybridation des provenances pures avec la provenance landaise se traduit par une augmentation de sensibilité, l'hybride se montrant plus sensible que chacune des 2 provenances "parentes". Cet effet de l'hybridation s'ajoute à l'effet provenance. L'hybridation se traduit également par une vigueur et une précocité accrues, l'hybride étant plus vigoureux et précoce que chacune des 2 provenances. L'augmentation de précocité liée à l'hybridation explique pour l'essentiel la plus grande sensibilité des hybrides. En effet, la sensibilité moyenne des hybrides n'est plus significativement différente de celle des provenances pures lorsque les données sont ajustées à la note moyenne de phénologie.

D'une façon générale, pour ce qui concerne les liaisons entre sensibilité et croissance (tableaux VIII et IX), la phénologie apparaît comme le caractère le plus explicatif du taux d'attaque, suivi par la longueur de la pousse apicale observée la même année. Le fait que la longueur de la pousse observée une année donnée ne soit une variable explicative significative que du taux d'attaque constaté la même année peut s'expliquer par une éventuelle interaction provenance $x$ année pour la croissance. Une interaction provenance $x$ station pourrait également être envisagée. Une étude multistationnelle et pluriannuelle est en cours afin de préciser ces aspects.

Sur un plan pratique, aucune race géographique n'apparaît significativement plus résistante à la rouille courbeuse que la race landaise. Le faible taux d'infection glo- 
bal du dispositif, dû à un développement insuffisant des trembles, pourrait toutefois masquer des différences. Les hybrides interraciaux sont d'autre part, dans l'ensemble, très sensibles, en tout cas au moins autant que la race landaise, même avec un "parent" plus résistant que celleci.

L'utilisation immédiate des races géographiques de pin maritime ne permet donc pas de créer de variétés résistantes à la rouille courbeuse. À l'inverse, on peut craindre des risques d'infection accrus avec l'utilisation d'hybrides. Ce dernier point devra être pris en considération dans une optique générale de lutte intégrée (faisant appel à plusieurs méthodes de lutte) en sylviculture intensive (utilisant notamment du matériel génétiquement amélioré).

Une telle conclusion ne présage pas de la possibilité de trouver dans certaines provenances quelques individus exceptionnels pour certains facteurs intervenant dans la résistance à $M$ pinitorqua. II peut s'agir de caractéristiques phénologiques, morphologiques ou biochimiques. Notre étude ne concerne en effet que le comportement moyen des provenances et devra être complétée, pour la recherche de génotypes rares, par l'utilisation d'autres dispositifs.

\section{RÉFÉRENCES}

Baradat P (1989) Amélioration génétique des arbres forestiers, éléments méthodologiques. Doc Laboratoire amélioration des arbres forestiers, INRA, Bordeaux

Baradat P, Marpeau-Bezard A (1988) Le pin maritime Pinus pinaster Ait. Biologie et génétique des terpènes pour la connaissance et l'amélioration de l'espèce. Thèse de doctorat d'État, université Bordeaux I

Baradat P, Pastuszka P, Durel CE (1990) Les hybrides entre races géographiques de pin maritime. For Gasc 364, 10
Debazac EF (1966) Les modalités de la croissance en hauteur chez les pins. Bull Soc Bot $F r, 3-14$

Desprez ML (1980) Melampsora pinitorqua. II. Partie expérimentale. Mémoire de DEA, INA, Paris-Grignon

Desprez-Loustau ML (1990) Intensification de la sylviculture et rouille courbeuse du pin maritime. In : Actes $3^{e}$ Coll Sci Ind bois, Arbora, Bordeaux, 14-15 mai, tome II, 523-527

Guyon JP (1980) Variabilité géographique et écophysiologique du pin maritime (Pinus pinaster Ait). Mémoire de fin d'études ENITEF, Nogent-sur-Vernisson

Guyon JP (1982) Croissance en hauteur et comportement hydrique de sept provenances de pin maritime (Pinus pinaster Ait) en plantations comparatives et en milieu contrôlé. Mémoire de DEA, université Bordeaux II

Kremer A, Roussel G (1986) Décomposition de la croissance en hauteur du pin maritime (Pinus pinaster Ait). Variabilité géographique des composantes morphogénétiques et phénologiques. Ann Sci For 43, 15-34

Kurkela T (1974) Communication. In : Compte rendu de la réunion du groupe S2 05-05 de I'IUFRO, Florence

Legendre L, Legendre P (1984) Écologie numérique (Tomes $1 \& 2$ ). $2^{e}$ édn, Masson, Paris

Longo N, Moriondo F, Naldini-Longo B (1980) Some aspects of biology of Melampsora pinitorqua Rostr in Italy, also compared to other European countries. Phytopathol Mediterr 19, 30-34

Petit R (1988) Ressources génétiques du pin maritime : apport des marqueurs enzymatiques. Mémoire DEA, Univ Paris-Sud

Philoche JL (1977) Une condition de validité pour le test $F$. Bull Assoc Statist Univ 1, 3759

Powers HR, Schmidt RA, Snow GA (1981) Current status and management of fusiform rust on southern pines. Ann Rev Phytopathol 19, 353-371

Sarrauste N (1982) Photosynthèse, respiration et production de matière sèche de jeunes plants de pin maritime (Pinus pinaster Ait) appartenant à sept provenances et conduits selon deux traitements hydriques. Mémoire de DEA, université Paris VII

Searle SR (1971) Linear models. Wiley, New York 
Sneath PHA, Sokal RR (1973) Numerical taxonomy - the principles of numerical classification. Freeman, San Francisco

Snedecor GW, Cochran WG (1967) Statistical methods (6th edition). The lowa State Univ, Ames, IA, USA

Sueron C (1982) Aspects génétiques et biochimiques de la résistance de Pinus pinaster à
Dioryctria sylvestrella. Mémoire ENITEF, Nogent-sur-Vernisson

Ughetto F (1981) Observations sur l'incidence en région méditerranéenne de Matsucoccus feytaudi Duc 1942 (Coccoidea, margarodidae) sur pins maritimes de diverses provenances. Mémoire de fin d'études, ENITEF, Nogent-sur-Vernisson 